to rectal mucosa in the receptive partner provides opportunity for the transmission of HIV/STI. The risk is more if the receptive partner is female. The aim of this study was to observe the prevalence and associated factors associated with anal sex practises among female sex workers (FSWs) in the western part of Assam, India.

Methods All total 64 FSWs were interviewed with a questionnaire to collect information regarding the respondents' knowledge and their practises related to anal sex as well as related socioeconomic factors.

Results 61 FSWs (95.3\%) reported having ever engaged in anal sex. $75 \%$ FSWs (48 cases) were aware of HIV/AIDS. Only 10.9\% FSWs (seven cases) were aware of the fact that unprotected anal sex has the higher risk of HIV transmission.

Conclusion This study showed the practise of anal sex is quite common among FSWs, but their perception of HIV transmission risk associated with anal sex is very low. This is an alarming situation. It is important for HIV prevention programmes to focus not only on vaginal sex but also on risk associated with anal sex.

\section{P4.028 CHILD MARRIAGE AND SEXUALLY TRANSMITTED INFECTIONS: IMPLICATIONS FOR HIV PREVENTION AMONG YOUNG MOTHERS IN ADAMAWA STATE NIGERIA}

doi:10.1136/sextrans-2013-051184.0926

E 0 Adeyemi. Lagos State University, Ojo, Lagos, Nigeria

In Nigeria, the practise of child marriage is deeply entrenched in culture and the country has one of the highest rates of child marriage in the world. The risks associated with early marriages are many especially among young girls, these include domestic violence, forced sexual act and increased vulnerability to Sexually Transmitted Infections (STIs). The study therefore examines the impact of child marriage on the spread of STIs and its implications for HIV infections.

The study was conducted in Gombi LGA in Adamawa State. Qualitative and quantitative methods were used for data gathering. Stratified sampling technique was employed to select the sample for the study. Two hundred young women aged 15-24 years who married before age 16 years were interviewedand 6 FGDs were conducted. Data collected were subjected to basic demographic analytical techniques and supported with qualitative data.

The median age of first marriage of the respondents is 15.5 years and $60 \%$ had primary school education. Sixty-two percent reported having diagnosed of at least one type of STIs. Treatments of STIs were more often than not outside government hospitals and clinics (77.5\%). Only $25 \%$ of the respondents ever discussed the need to use condoms with their husbands even when they are at risks. There is a significant relationship $(\mathrm{P}<0.001)$ between age, sexual negotiations, level of education, use of condoms and ever contacted STIs

In the context of widespread STIs and growing evidence of HIV/ AIDS against women within permissive traditional practises, the health implications of early marriage as practised in Gombi are grave for Nigeria. There is need to focus on the cultural traps to which the practise of child marriage has confined girls in Gombi and other parts of the country, through renewed commitment to compulsory education beyond primary school level for girls.

\section{P4.029 CONCURRENT SEXUAL PARTNERSHIPS IN A NATIONAL SAMPLE OF AUSTRALIAN GAY MEN}

doi:10.1136/sextrans-2013-051184.0927

'A Lyons, ${ }^{2}$ W Hosking. 'La Trobe University, Melbourne, Australia; ${ }^{2}$ Victoria University, Melbourne, Australia
Concurrent sexual partnerships, where individuals have two or more sexual partners at the same time, are major contributors to the transmission of sexually transmitted infections (STIs). Yet, very little is known about the prevalence and predictors of sexual concurrency in populations of men who have sex with men. This presentation reports on the findings of a national community-based survey of 1,049 Australian gay men aged 18-39 years, who were asked about their sexual partners over a 12-month period. Of those who reported having sex in the past 12 months ( $N=901), 28 \%$ reported at least one period in which they had concurrent sexual partners. Of this group, $57 \%$ had sex concurrently with two partners, $25 \%$ with three partners, and $18 \%$ with four or more partners. Worryingly, 64\% reported having unprotected sex with one or more of their concurrent partners. A multivariate logistic regression found sexual concurrency was just as likely among men of all ages. However, sexual concurrency was significantly more prevalent among those on higher incomes $(\mathrm{P}=0.01)$, who were not in an ongoing relationship $(\mathrm{P}=0.009)$, and who reported having large numbers of sexual partners in the past 12 months $(\mathrm{P}<0.001)$. Of men who were tested for an STI in the past 12 months, STIs were more prevalent among those who reported sexual concurrency $(20 \%$ vs. $13 \%, \mathrm{P}=0.04)$. In all, concurrent sexual partnerships appear to be common among 18-39 year old gay men in Australia. Discussion will focus on ways in which these data provide information for understanding and controlling STI epidemics in populations of gay men and other men who have sex with men.

\section{P4.030 FACTORS INFLUENCING CONDOM NON-USE IN TURKS \& CAICOS ISLANDS}

doi:10.1136/sextrans-2013-051184.0928

${ }^{1}$ A M Robinson, ${ }^{2} Y$ Holder, ${ }^{3} \mathrm{~T}$ E Maitland. ${ }^{1}$ National AIDS Programme, Grand Turk, Turks and Caicos Islands, ${ }^{2}$ Consultant Epidemiologist/Biostatistician, Castries, Saint Lucia, ${ }^{3}$ National Epidemiology and Research Unit, Grand Turk, Turks and Caicos Islands

Background The Turks and Caicos Islands (TCI) is a UK overseas territory in the Caribbean, with an estimated population of 35,000. In 2003/2004, the National AIDS Programme became fully operational, offering voluntary counselling and treatment, in addition to other efforts to prevent the spread of HIV/AIDS. In 2010, the European Union commissioned this study to assess current knowledge, attitudes and risk practises for HIV in the Turks and Caicos Islands, after the Programme's decade of operation.

Methods A KABP questionnaire was administered to a random sample of 837 persons representative of the territory's 15-49 year old population and the data generated was manipulated and analysed using SPSS.

Results $92.1 \%$ of respondents were sexually active and overwhelmingly heterosexual (97.7\%). Of these, more than half $(54.1 \%)$ did not use condoms, with percentage non-use increasing with age. Factors significantly associated with condom non-use were number of partners, living arrangements, age and sex while under the influence of alcohol or drugs.

$62.8 \%$ of those not using condoms, gave "trust my partner" as their reason, yet $65 \%$ of trusting partners tested within the past year. Further, $58 \%$ of all respondents were unsure of their partner's reaction to using a condom, feared that their partner would be upset and/or were embarrassed at the use of a condom.

Conclusion These data speak to a dire need for condom negotiating skills among older persons, reputedly in committed relationships, especially given that one in nine had multiple partners. The challenge is to employ a strategy that is appropriate to and acceptable by such a population. One possibility is to promote condom use as a sign of respect and care for one's partner. 